\title{
BreastCare
}

\section{Systemic Treatment in Premenopausal Patients with Breast Cancer}

\author{
Georg Pfeiler \\ Deptartment of Gynecology and Gynecologic Oncology, Medical University of Vienna, Austria
}

In young, premenopausal women, diagnosis and therapy of breast cancer is a challenge in daily clinical routine. Host and tumor strikingly differ from the postmenopausal situation. Besides clinical examination of the breast, no early detection program is established in young women - except for women carrying a BRCA mutation. Because of the higher density of the breasts, routine mammographic controls are not useful for early detection in young women, and magnetic resonance imaging (MRI) - which clearly overcomes this problem and is used for screening in high-risk BRCA mutation carriers - has limited access, needs high expertise and has to be performed according to the menstrual cycle. The missing early detection is at least in part responsible for the higher tumor stage in young patients. Menstrual cycle might not only impact on detection but also on efficacy of therapy and outcome, even though Grant et al. [1] elegantly demonstrated that this is not true for surgical treatment of breast cancer. Other factors which obviously show differences due to menopausal status are known. High body mass index (BMI) clearly increases breast cancer risk in postmenopausal women, whereas it seems to have no impact or even leads to risk reduction in premenopausal women. Recently, Dowsett et al. [2] hypothesized that this inverse relation of BMI and breast cancer risk in premenopausal women may be due to reduced progesterone serum levels in obese women. Conversely it has been shown that BMI impacts breast cancer outcome especially in premenopausal but not postmenopausal women $[3,4]$.

In young patients with breast cancer, co-morbidities are rare and usually do not limit use of systemic treatment options. Even more, life expectancy is high and somewhat forces to use every possible treatment option. This is of course a psychological problem as well - from the physicians and patients point of view, respectively. However, it is not always necessary to use every conceivable therapy modality in young, premenopausal patients with breast cancer: In the ABCSG-12 trial, premenopausal patients with breast cancer received goserelin and were randomized to anastrozole versus tamoxifen $+/$ - zoledronic acid. Even though one third of the patients had nodal positive disease only $6 \%$ received chemotherapy. The ac- tive treatment period for endocrine therapy was 3 years. With a median follow up of 48 months, a $97 \%$ overall survival could be demonstrated for these premenopausal patients [5].

The improvements in breast cancer outcomes in younger women as well as the per se high life expectancy in this collective demand to thoroughly consider treatment side effects - especially long-term side effects. Chemotherapy generally impairs ovary function throughout the active treatment period but also reduces the ovary reserve, leading to an early decline of serum estrogen levels and early menopause. This impact of chemotherapy on the ovary leads to reduced fertility, which needs to be addressed especially in young patients who have not yet completed family planning. Good data exist that pregnancy after breast cancer does not increase risk of recurrence [6]. Therefore fertility concerns need to be discussed before starting systemic treatment for breast cancer. This is especially important as new data demonstrated that down regulation of ovary function before and during chemotherapy reduces post-therapeutic amenorrhoea and increases pregnancy rates [7]. Both, chemotherapy and endocrine treatment strikingly impact on bone health in premenopausal patients with breast cancer. Reduced estrogen serum levels lead to dramatic declines in bone mineral density and increase fracture risk. Having in mind the important findings of the SOFT and TEXT trial, osteoporosis will be the major issue for young breast cancer survivors.

Not only the host differs: We have good evidence that breast cancer in young, premenopausal women shows a more aggressive phenotype. Breast cancers of younger women tend to be more often hormone receptor negative, G3, Her2-neu positive and diagnosed in a higher stage compared to postmenopausal breast cancer. This more aggressive behavior of the tumor often demands cytotoxic treatment regimens. In this special focus issue Ann H. Patridge elegantly summarizes the indications, cytotoxic treatment options and side effects of treatment for young, premenopausal patients with breast cancer. Besides chemotherapy $+/$ - Her2-targeted therapy, endocrine treatment is the second important backbone of systemic treatment in premenopausal patients with breast

\section{KARGER}

Fax +497614520714
() 2015 S. Karger GmbH, Freiburg

$1661-3791 / 15 / 0105-0305 \$ 39.50 / 0$ assoz. Prof. PD Dr. Georg Pfeiler

Abteilung für Gynäkologie und gynäkologische Onkologie

Medizinische Universität Wien, AKH Wien

Währinger Gürtel 18-20, 1090 Wien, Austria

georg.pfeiler@meduniwien.ac.at 
cancer. Especially, findings of the SOFT and TEXT trials but also long-term endocrine therapy data lead to a variety of endocrine treatment options, which have to be intensively discussed with the patient. In this special focus issue Lorenzo Rossi and Olivia Pagani discuss the endocrine therapy as well as its side effects and propose a treatment decision flow chart for endocrine therapy in premenopausal women with hormone receptor positive breast cancer.

\section{Disclosure Statement}

The author has no conflicts of interest to disclose.

\section{References}

1 Grant CS1, Ingle JN, Suman VJ, Dumesic DA, Wickerham DL, Gelber RD, Flynn PJ, Weir LM, Intra M Jones WO, Perez EA, Hartmann LC: Menstrual cycle and surgical treatment of breast cancer: findings from the NCCTG N9431 study. J Clin Oncol 2009;27:36203626 .

2 Dowsett M, Folkerd E: Reduced progesterone levels explain the reduced risk of breast cancer in obese premenopausal women: a new hypothesis. Breast Cancer Res Treat 2015;149:1-4.

3 Pfeiler G, Königsberg R, Hadji P, Fitzal F, Maroske M Dressel-Ban G, Zellinger J, Exner R, Seifert M, Singer C, Gnant M, Dubsky P: Impact of body mass index on estradiol depletion by aromatase inhibitors in postmenopausal women with early breast cancer. Br J Cancer 2013;109:1522-1527.
4 Pan H, Gray RG; on behalf of the Early Breast Cancer Trialists' Collaborative Group: Effect of obesity in premenopausal ER+ early breast cancer: EBCTCG data on 80,000 patients in 70 trials. J Clin Oncol 2014;32(suppl): 5-s (Abstr 503).

5 Gnant M, Mlineritsch B, Schippinger W, LuschinEbengreuth G, Pöstlberger S, Menzel C, Jakesz R, Seifert M, Hubalek M, Bjelic-Radisic V, Samonigg H, Tausch C, Eidtmann H, Steger G, Kwasny W, Dubsky P, Fridrik M, Fitzal F, Stierer M, Rücklinger E, Greil R; ABCSG-12 Trial Investigators, Marth C: Endocrine therapy plus zoledronic acid in premenopausal breast cancer. N Engl J Med 2009;360:679-691.
6 Azim HA Jr, Kroman N, Paesmans M, Gelber S, Rotmensz N, Ameye L, De Mattos-Arruda L, Pistilli B Pinto A, Jensen MB, Cordoba O, de Azambuja E, Goldhirsch A, Piccart MJ, Peccatori FA: Prognostic impact of pregnancy after breast cancer according to estrogen receptor status: a multicenter retrospective study. J Clin Oncol 2013;31:73-79.

7 Moore HC, Unger JM, Phillips KA, Boyle F, Hitre E, Porter D, Francis PA, Goldstein LJ, Gomez HL, Vallejos CS, Partridge AH, Dakhil SR, Garcia AA, Gralow J, Lombard JM, Forbes JF, Martino S, Barlow WE, Fabian CJ, Minasian L, Meyskens FL Jr, Gelber RD, Hortobagyi GN, Albain KS; POEMS/S0230 Investigators: Goserelin for ovarian protection during breast-cancer adjuvant chemotherapy. N Engl J Med 2015;372:923932. 